\title{
Consolidation of $\left[\left(\mathrm{Fe}_{0.5} \mathrm{Co}_{0.5}\right)_{0.75} \mathrm{Si}_{0.05} \mathrm{~B}_{0.2}\right]_{96} \mathrm{Nb}_{4}$ Metallic Glassy Powder by SPS Method*1
}

\author{
N. Yodoshi*2, R. Yamada*2, A. Kawasaki and R. Watanabe*3 \\ Department of Materials Processing, Graduate School of Engineering, Tohoku University, Sendai 980-8579, Japan
}

Metallic glasses have been reported to exhibit excellent properties, such as high strength, high corrosion resistance, high wear resistance, which result from their amorphous structure. Because of a drastic reduction in their viscosities in supercooled liquid region, metallic glasses have an excellent workability through viscous deformation for the production of various industrial parts.

Recently, much attention has been paid to Fe-based metallic glasses because of their rich resources in addition to their excellent mechanical and magnetic properties. However, due to their poor glass-forming ability, the size of the Fe-based bulk metallic glass by conventional casting techniques is limited. In the present investigation, nearly fully densified disk-shaped compacts of $\left[\left(\mathrm{Fe}_{0.5} \mathrm{Co}_{0.5}\right)_{0.75} \mathrm{Si}_{0.05} \mathrm{~B}_{0.2}\right]_{96} \mathrm{Nb}_{4}$ metallic glass were produced from a gas-atomized amorphous powder by spark plasma sintering (SPS). The processing temperature and the time that assure the supercooled liquid state of the compacts were determined from the Time-Temperature-Transformation (TTT) diagram that was constructed by isothermal differential scanning calorimetry. The mechanical properties of the consolidated samples were evaluated by compression test. Comparatively low values of the fracture stress, Young's modulus and yielding stress of SPSed sample compared to the casted samples were observed, which is discussed on the basis of the integrity of the interparticle bonding. [doi:10.2320/matertrans.M2009093]

(Received April 1, 2009; Accepted June 19, 2009; Published August 5, 2009)

Keywords: powder consolidation, bulk metallic glass, metallic glassy powders, spark plasma sintering, densification control

\section{Introduction}

Fe-Co based metallic glasses exhibit high strength exceeding $4000 \mathrm{MPa}$ and have a merit of low cost owing to rich resources. ${ }^{1)}$ Among various metallic glasses under investigation for practical applications, the Fe-Co based glassy alloys are expected, in particular, to be advanced structure materials. However, the glass forming ability, as well as the thermal stability of the supercooled liquid, of the $\mathrm{Fe}-\mathrm{Co}$ based glassy alloys are low compared to $\mathrm{Zr}^{2}{ }^{2,3)}$ and $\mathrm{Pd}_{-}{ }^{4}$ based metallic glasses. It requires a cooling rate of over $10^{3} \mathrm{~K} / \mathrm{s}$ to produce $\mathrm{Fe}-\mathrm{Co}$ based bulk metallic glasses. Bulk samples of this alloy of the dimension of over $10 \mathrm{~mm}$ cannot be produced by conventional copper mould casting techniques. ${ }^{5,6)}$ Powder metallurgy would be the alternative to the casting process in the production of the Fe-Co based bulk metallic glasses with large dimensions and complex shapes. ${ }^{7)}$ Because of poor plasticity, metallic glasses are hard to be consolidated by conventional die compaction at room temperature. But they deform easily after the glass transition with drastic reduction in viscosity ${ }^{8)}$ at temperatures far below the usual sintering temperature $\left(\sim 0.7 \mathrm{~T}_{\mathrm{m}}\right)$. In this context, pressurized hot consolidation is considered to be one of the most suitable methods for the production of the bulk compacts. Here, we should note that they crystallize in a certain incubation time when held at high temperatures even if it is in the glass state. Therefore, it is indispensable to know the incubation time for crystallization, during which powder compacts can be consolidated in keeping a glassy state. The information on the incubation time is obtained from the Time-Temperature-Transformation (TTT) curve, which is usually constructed from isothermal DSC analyses at various

\footnotetext{
*1 This Paper was Originally Published in Japanese in J. Japan Inst. Metals 73 (2009) 299-305.

${ }^{* 2}$ Graduate Student, Tohoku University

${ }^{* 3}$ Professor Emeritus, Tohoku University
}

temperatures. Considering a limited processing time the spark plasma sintering (SPS) method is one of the best choices among various hot consolidation processes because it enables a short consolidation time and in addition to provide an possibility of removing surface oxide films of powder particles. ${ }^{9,10)}$ It is reported that Ni-based bulk metallic glasses with nearly full density have been obtained by the SPS, and that their compressive fracture strength is comparative to those of cast materials of the same composition. ${ }^{9}{ }^{\text {) }}$

In the present paper we report the SPS consolidation of a gas-atomized metallic glassy powder of $\left[\left(\mathrm{Fe}_{0.5} \mathrm{Co}_{0.5}\right)_{0.75^{-}}\right.$ $\left.\mathrm{Si}_{0.05} \mathrm{~B}_{0.2}\right]_{96} \mathrm{Nb}_{4}$ alloy system that shows a comparatively high glass forming ability and high strength property. ${ }^{5,6,11)}$ The consolidated compacts are evaluated by compressive test at room temperature.

\section{Experimental Procedures}

Master ingots of the $\left[\left(\mathrm{Fe}_{0.5} \mathrm{Co}_{0.5}\right)_{0.75} \mathrm{Si}_{0.05} \mathrm{~B}_{0.2}\right]_{96} \mathrm{Nb}_{4}$ alloy were prepared by arc melting of a mixture of high purity Fe, $\mathrm{Co}, \mathrm{Si}, \mathrm{B}$ and $\mathrm{Nb}$ under an argon atmosphere. The metallic glassy powder was produced by a high pressure atomization. The melt was bottom-poured through a $3 \mathrm{~mm}$ nozzle into an annular gas atomizer at a pressure of $9.8 \mathrm{MPa}$ of argon. The atomized powder was sieved to under $125 \mu \mathrm{m}$. The glassy structure of the atomized powder and the absence of a micrometer-scale crystalline phase were confirmed by XRD and SEM, respectively. The thermal stability expressed by the glass transition temperature $T_{\mathrm{g}}$, crystallization temperature $T_{\mathrm{x}}$, and supercooled liquid region $\Delta T_{\mathrm{x}}\left(=T_{\mathrm{x}}-T_{\mathrm{g}}\right)$, was examined by differential scanning calorimetry (DSC) under pure argon at a heating rate of $0.67 \mathrm{~K} / \mathrm{s}$. The TTT diagram was constructed by the DSC data to assess the consolidation temperature and the time that assure the supercooled liquid state during consolidation. The atomized powder was consolidated by SPS with two kinds of $10 \mathrm{~mm}$ diam. dies. A carbon die was used under consolidation pressure of $75 \mathrm{MPa}$, 


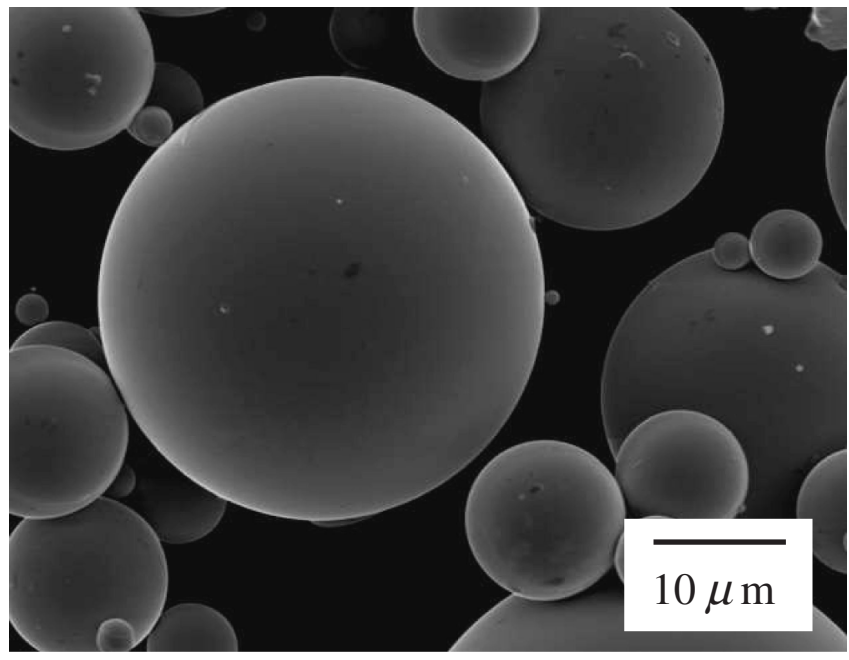

Fig. 1 SEM image of atomized $\left[\left(\mathrm{Fe}_{0.5} \mathrm{Co}_{0.5}\right)_{0.75} \mathrm{Si}_{0.05} \mathrm{~B}_{0.2}\right]_{96} \mathrm{Nb}_{4}$ metallic glassy powder.

and a hard metal die, $400 \mathrm{MPa}$. The consolidation temperature was controlled by pulsed direct current with thermocouples inserted into the dies. The heating rate was $0.5 \sim 0.67 \mathrm{~K} / \mathrm{s}$. The densities of the consolidated compacts were measured by Archimedes method. Test specimens of dimensions of $3 \times 3 \times 3 \mathrm{~mm}$ were prepared for compression test. The cross head speed of the compression test was $0.5 \mathrm{~mm} / \mathrm{min}$. The fracture surface of the test specimen was observed by SEM. TEM observation was also made to inspect the bonding state of the compressed particles. Low energy ion milling was used for the preparation of TEM samples to avoid ion-beam induced crystallization.

\section{Results and Discussion}

\subsection{Evaluation of gas atomized powder}

Figure 1 shows a SEM image of the gas-atomized $\left[\left(\mathrm{Fe}_{0.5} \mathrm{Co}_{0.5}\right)_{0.75} \mathrm{Si}_{0.05} \mathrm{~B}_{0.2}\right]_{96} \mathrm{Nb}_{4}$ alloy powder. The powder is spherical and composed of various sizes of particles. The particle surfaces are smooth and exhibit no contrast, showing homogeneous microstructure. The XRD pattern of the powder shown in Fig. 2 is a broad pattern peculiar to the amorphous structure, and appears to have no appreciable amount of crystalline phases. The DSC curve of the powder is shown in Fig. 3, in which an endothermic peak starts to appear at about $807 \mathrm{~K}$, and a sharp exothermic peak at $855 \mathrm{~K}$. The former peak is with glass transition and the latter with crystallization. The glass transition temperature $T_{\mathrm{g}}$, and onset temperature of crystallization $T_{\mathrm{x}}$, are determined to be 807.5 and $855.5 \mathrm{~K}$, respectively. The thermal stability $\Delta T$ is $48 \mathrm{~K}$. These values agree with the earlier data. ${ }^{5,6)}$

\subsection{Construction of TTT diagram}

Figure 4 shows a schematic diagram of the temperature schedule of the isothermal DSC analysis, showing an estimation of the incubation time. It was assumed in the present study that the zero time of the incubation is $700 \mathrm{~K}$, which was estimated from the transition temperature to a supercooled liquid state in this alloy system and regarded as the temperature of appreciable atomic movement for the

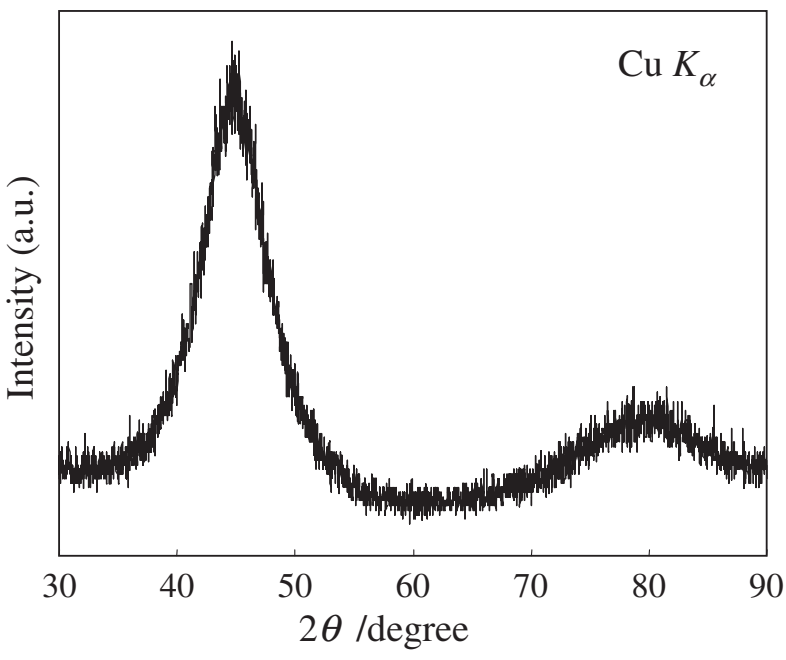

Fig. 2 XRD pattern of atomized $\left[\left(\mathrm{Fe}_{0.5} \mathrm{Co}_{0.5}\right)_{0.75} \mathrm{Si}_{0.05} \mathrm{~B}_{0.2}\right]_{96} \mathrm{Nb}_{4}$ metallic glassy powder.

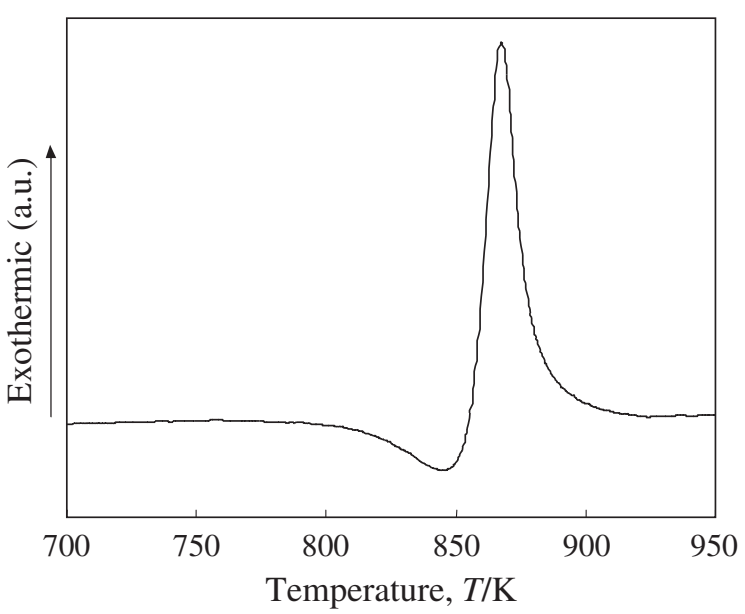

Fig. 3 DSC curve of atomized $\left[\left(\mathrm{Fe}_{0.5} \mathrm{Co}_{0.5}\right)_{0.75} \mathrm{Si}_{0.05} \mathrm{~B}_{0.2}\right]_{96} \mathrm{Nb}_{4}$ metallic glassy powder. (Heating rate: $0.67 \mathrm{~K} / \mathrm{s}, T_{\mathrm{g}}: 807.5 \mathrm{~K}, T_{\mathrm{x}}: 855.5 \mathrm{~K}$, $\left.\Delta T_{\mathrm{x}}\left(=T_{\mathrm{x}}-T_{\mathrm{g}}\right): 48.0 \mathrm{~K}\right)$

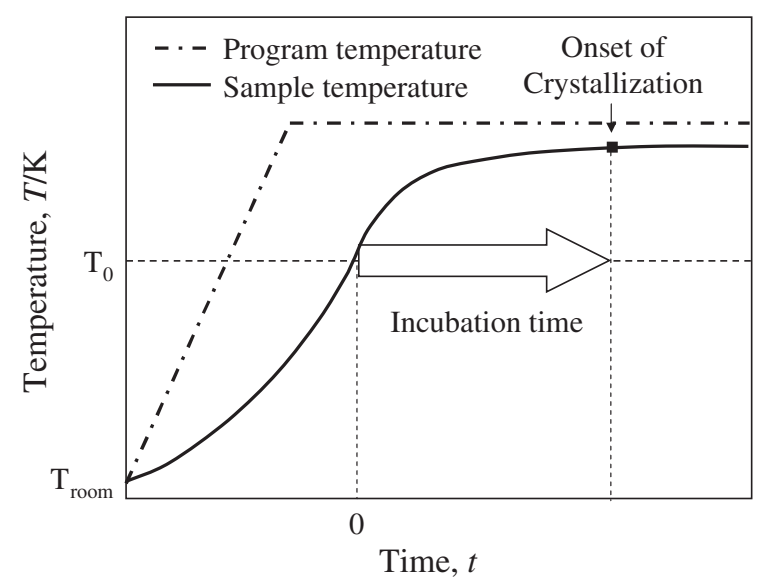

Fig. 4 Programmed and sample temperatures for isothermal DSC. $T_{0}$ is assumed to be $700 \mathrm{~K}$ in the present investigation. The definition of the incubation time for the crystallization is also shown. 


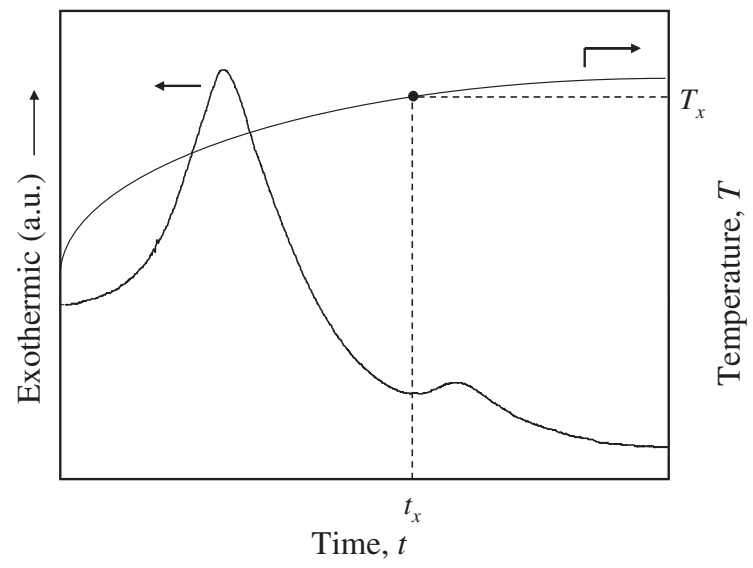

Fig. 5 The determination of the crystallization temperature $T_{\mathrm{x}}$ from DSC curve.

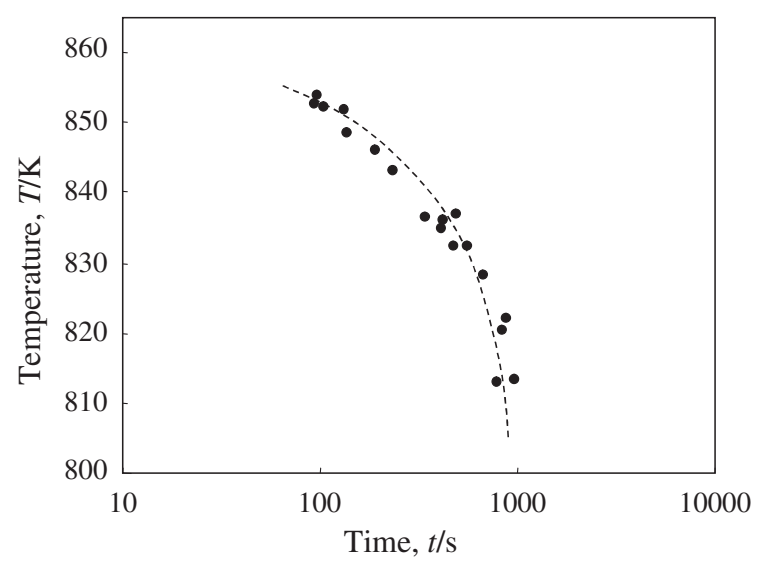

Fig. 6 TTT diagram for gas-atomized $\left[\left(\mathrm{Fe}_{0.5} \mathrm{Co}_{0.5}\right)_{0.75} \mathrm{Si}_{0.05} \mathrm{~B}_{0.2}\right]_{96} \mathrm{Nb}_{4}$ metallic glass. The time axis is expressed in logarithmic scale.

structural modification. It was confirmed that nano crystalline phases were formed by a heat treatment of $700 \mathrm{~K}$ for $10^{5} \mathrm{~s}$. The onset temperature of the crystallization was determined from the DSC analysis as the starting temperature of the exothermic peak, as shown in Fig. 5. The TTT curve thus obtained is shown in Fig. 6, where the incubation time for the crystallization is seen to decrease with increasing temperature. The incubation time at $T_{\mathrm{g}}$ and $T_{\mathrm{x}}$ are about $1000 \mathrm{~s}$ and $100 \mathrm{~s}$, respectively. As far as the sample temperatures are held within the times read off from this curve, the sample keeps glassy state and viscous deformation.

\subsection{Consolidation}

Table 1 summarizes the SPS consolidation data and the attained relative density of the compacts. The holding time was determined from the TTT curve to be within the incubation time. Figure 7 shows the relative density of the compacts as a function of holding temperature, together with the SEM images of the cross sections of some samples. The symbols (a) (f) attached to the data points and photos are those listed in Table 1. Steep increases in relative density against holding temperature are seen in this diagram. At the pressures of 75 and $400 \mathrm{MPa}$, the increases occur at about $760 \mathrm{~K}$ and $730 \mathrm{~K}$, respectively. Such critical behavior of the relative density would correspond to a drastic reduction
Table 1 Process-variables of the SPS-consolidation and the relative density of the consolidated compacts.

\begin{tabular}{cccccc}
\hline Sample & $\begin{array}{c}\text { Pressure } \\
(\mathrm{MPa})\end{array}$ & $\begin{array}{c}\text { Holding } \\
\text { temperature } \\
(\mathrm{K})\end{array}$ & $\begin{array}{c}\text { Holding } \\
\text { time } \\
(\mathrm{s})\end{array}$ & $\begin{array}{c}\text { Heating } \\
\text { rate } \\
(\mathrm{K} / \mathrm{s})\end{array}$ & $\begin{array}{c}\text { Relative } \\
\text { density } \\
(\%)\end{array}$ \\
\hline $\mathrm{a}$ & 75 & 753 & 180 & 0.67 & 94.6 \\
\hline $\mathrm{b}$ & 75 & 773 & 180 & 0.70 & 97.1 \\
\hline $\mathrm{c}$ & 75 & 833 & 60 & 0.55 & 99.0 \\
\hline $\mathrm{d}$ & 75 & 833 & 180 & 0.55 & 98.8 \\
\hline $\mathrm{e}$ & 400 & 763 & 120 & 0.62 & 99.6 \\
\hline $\mathrm{f}$ & 75 & 793 & 180 & 0.67 & 98.8 \\
\hline
\end{tabular}

in viscosity at these temperatures to enhance the viscous densification. The sample (c) of the relative density of $99.0 \%$ consolidated at the pressure of $75 \mathrm{MPa}$ for $60 \mathrm{~s}$ at $833 \mathrm{~K} \mathrm{kept}$ amorphous state as confirmed by XRD, while the sample (d) held $180 \mathrm{~s}$ at the same temperature crystallized. At the temperature of $848 \mathrm{~K}$, the crystallization was found to occur in $60 \mathrm{~s}$. The microstructure evolution during densification can roughly be seen in the SEM images given in Fig. 7. The photograph (a) shows the compact of the relative density of 94.6\%; densification is insufficient and typical polyhedral deformation of the particles is clearly seen, but the interparticle bonding seems still weak. With densification interparticle contacts become more intimately bonded (see photo (b)), and in the sample (c) of the relative density of $99.0 \%$, the interparticle contacts seem to be well bonded. The maximum relative density of $99.6 \%$ was attained under the pressure of $400 \mathrm{MPa}$, for $120 \mathrm{~s}$ at $763 \mathrm{~K}$. It is to be noted that the shape of the pores is cusped throughout the consolidation process. The concave pore surface usually observed in sintering of metal powders does not appear in the present case. This is probably because the deformation proceeds mainly by viscous flow under the applied stress, and the temperature and the time are not sufficient for surfacetension derived spontaneous morphological relaxation.

\subsection{Crystallization behavior during consolidation}

Figure 8 shows the XRD patterns of the compacts (c) and (d), both were consolidated under the same pressure and temperature $(75 \mathrm{MPa}$ and $833 \mathrm{~K})$, but for different holding times. Although two patterns are basically of amorphous nature, for the compact (d) peaks of a metastable crystalline phase, $(\mathrm{Fe}, \mathrm{Co}, \mathrm{Nb})_{23} \mathrm{~B}_{6}$, though weak, can be discerned. The crystallization behavior in the present glassy alloy system will be discussed more in detail on the basis of the TTT curve and the heating program, both shown in Fig. 9. The heating curve for the compact (c) lies under the TTT curve throughout the consolidation process, while that for the compact (d) crosses the TTT curve at about $450 \mathrm{~s}$. This indicates, together with the change in XRD patterns described above, that the compact (c) kept amorphous state during heating, but in the compact (d), the crystallization took place, if any, during consolidation.

\subsection{Mechanical properties}

Figure 10 shows the compressive stress-strain curve for the compact (c). From this curve the fracture strength and 

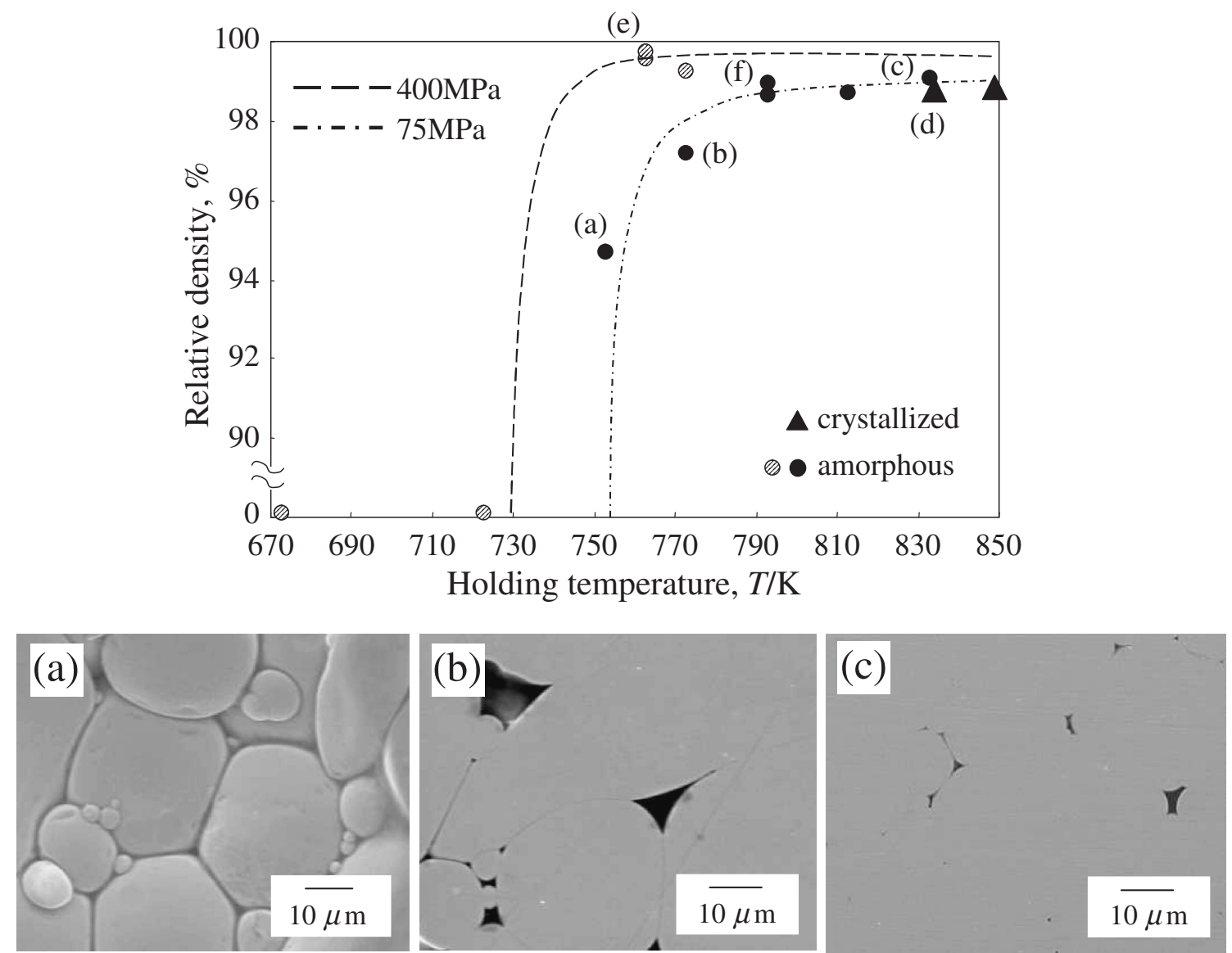

Fig. 7 Relation between relative density and holding temperature, and SEM images of the cross sections of some selected samples for $\left[\left(\mathrm{Fe}_{0.5} \mathrm{Co}_{0.5}\right)_{0.75} \mathrm{Si}_{0.05} \mathrm{~B}_{0.2}\right]_{96} \mathrm{Nb}_{4}$ metallic glassy powder compacts. Symbols (a), (b), .., (f) attached to the data points and the photos are listed in Table 1.

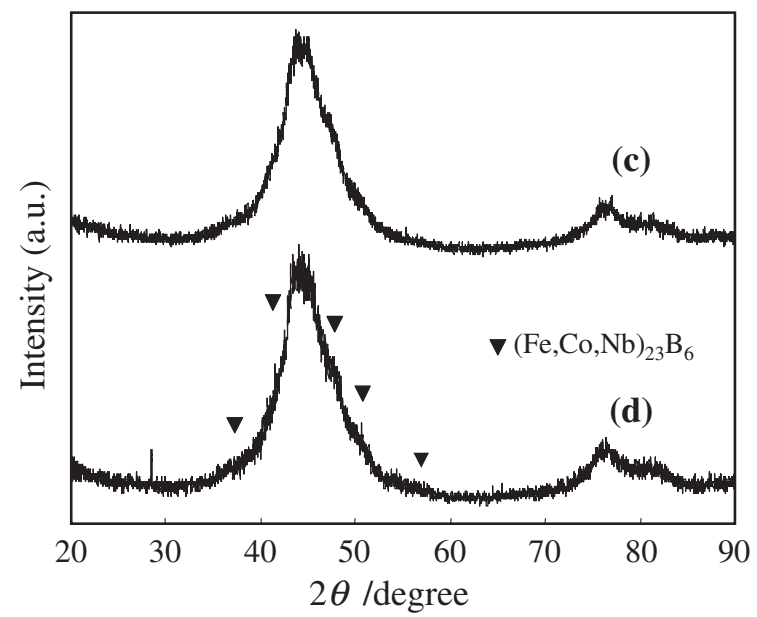

Fig. 8 XRD patterns of the samples (c) and (d), SPS-consolidated for $60 \mathrm{~s}$ and $180 \mathrm{~s}$, respectively, at the pressure of $75 \mathrm{MPa}$ and the temperature of $833 \mathrm{~K}$.

Young's modulus of the present metallic glassy alloy are estimated to be $900 \mathrm{MPa}$ and $194 \mathrm{GPa}$, respectively. It has been reported that the compressive fracture strength of the metallic glassy alloy of the same composition produced by copper mold casting method is over $4000 \mathrm{MPa} .^{5,6)}$ The yield strain of the present alloy is about $4.6 \times 10^{-3}$, which is also lower than those $\left(\sim 2 \times 10^{-2}\right)$ reported for the casted metallic

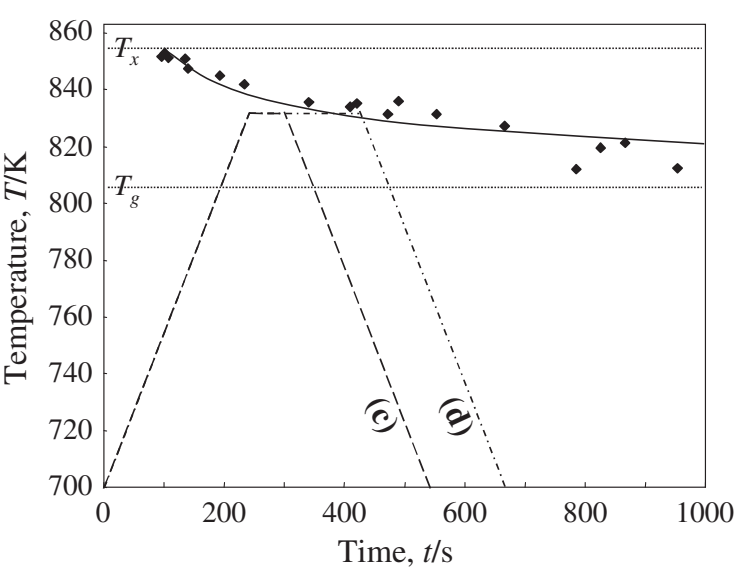

Fig. 9 Temperature programs of the SPS-consolidation, together with the TTT diagram for the $\left[\left(\mathrm{Fe}_{0.5} \mathrm{Co}_{0.5}\right)_{0.75} \mathrm{Si}_{0.05} \mathrm{~B}_{0.2}\right]_{96} \mathrm{Nb}_{4}$ metallic glassy powder compacts. The glass transition and crystallization temperatures are also shown.

glasses. ${ }^{12)}$ The fracture surface of the test sample is shown in Fig. 11. Although intragranular fracture with a kind of vein structure is seen in some grains, intergranular fracture is prevailing throughout the sample. In fact, our additional bending experiment on the same sample, where fracture occurs by tensile stress, shows complete intergranular fracture. These observations suggest that the bonding 


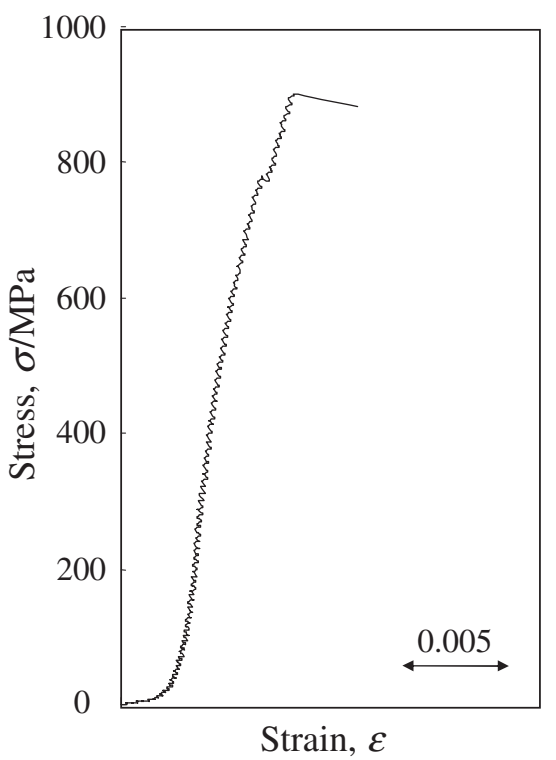

Fig. 10 Compressive stress-strain curve of the SPS-consolidated for $60 \mathrm{~s}$ at the pressure of $75 \mathrm{MPa}$ and the temperature of $833 \mathrm{~K}$. The sample dimensions are $3.06 \times 3.52 \times 6.42 \mathrm{~mm}$.

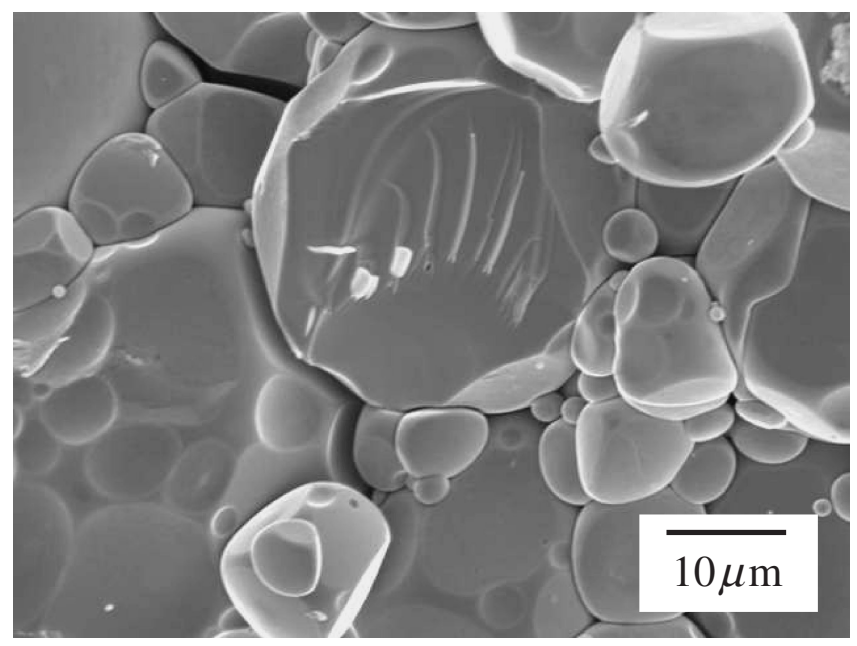

Fig. 11 SEM image of the fracture surface of the compression test sample, SPS-consolidated for $60 \mathrm{~s}$ at the pressure of $75 \mathrm{MPa}$ and the temperature of $833 \mathrm{~K}$.

strength of the grain interface of the present sample is weak. The TEM image of the sample of $98.8 \%$ relative density (sample (f)) is shown in Fig. 12. The electron diffraction pattern is from the inside of the particle, and a continuous ring shows that the particles are amorphous. Note that the crystallized phase of a thickness of about $50 \mathrm{~nm}$ is seen in the bonded region of the particles. This is presumably a main cause of the bonding weakness.

Little work has been done on the viscous flow behavior and the hot consolidation of metallic glass powders of the present composition. The nature of the bonding of individual particles of metallic glass in an atomic scale during hot consolidation is still not fully clarified. In particular, processing conditions, as well as the mechanism, of the formation of crystalline phase at the interparticle bonded region is of great importance, since it seems to determine the fracture strength of the consolidated compacts.

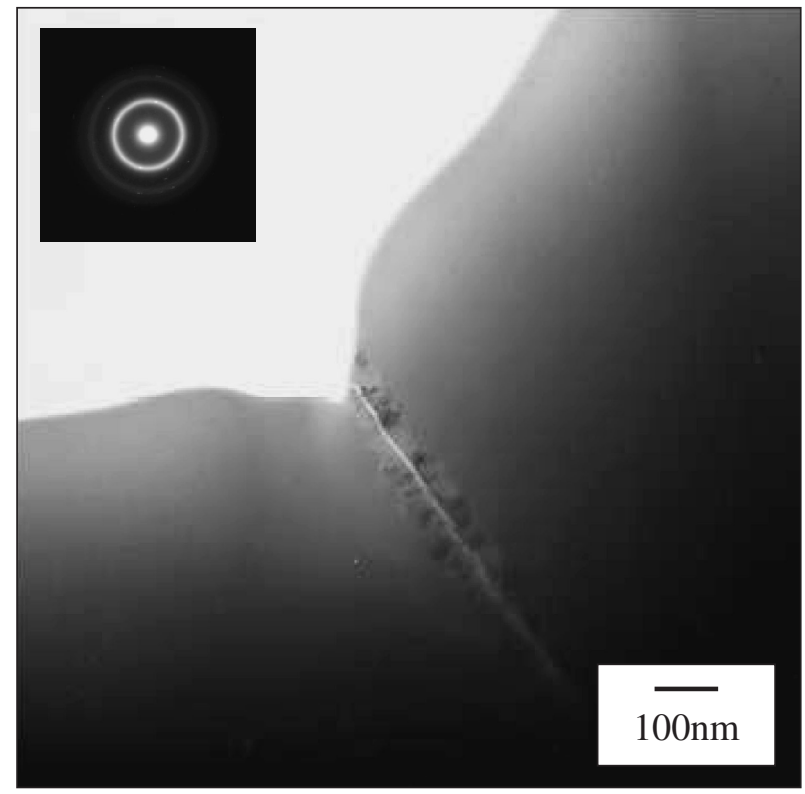

Fig. 12 TEM image of the bonded region of the sample SPS-consolidated for $180 \mathrm{~s}$ at the pressure of $75 \mathrm{MPa}$ and the temperature of $793 \mathrm{~K}$. The diffraction pattern from the inside of the particle is shown. The crystallized layer of the thickness of about $50 \mathrm{~nm}$ is seen.

\section{Conclusion}

(1) The atomized powder of $\left[\left(\mathrm{Fe}_{0.5} \mathrm{Co}_{0.5}\right)_{0.75} \mathrm{Si}_{0.05} \mathrm{~B}_{0.2}\right]_{96^{-}}$ $\mathrm{Nb}_{4}$ metallic glass can be consolidated into a diskshaped compact of $99.6 \%$ relative density having a diam. of $10 \mathrm{~mm}$ by SPS method.

(2) There are critical temperatures for the densification curve to show steep increase, which depends on the applied pressure. The critical temperatures are $740 \mathrm{~K}$ and $760 \mathrm{~K}$ at the pressure of $400 \mathrm{MPa}$ and $75 \mathrm{MPa}$, respectively. Such rapid change in densification is supposed to depend on the viscosity change, which in turn depends on temperature.

(3) The shape of pores is kept cusped throughout the consolidation, indicating that no surface relaxation due to surface tension takes place during hot consolidation, presumably because of comparatively low temperature and short consolidation time. The densification in the present case occurs mainly by a viscous deformation of particle assemblage under applied pressure.

(4) The fracture strength, Young's modulus and yielding strain of the present metallic glass alloy as estimated by compression test are about $900 \mathrm{MPa}, 194 \mathrm{GPa}$ and $4.6 \times 10^{-3}$, respectively. These values are comparatively low compared to those reported for the conventional casted samples, and these low values are to be attributed to the presence of a thin crystallized layer at the interparticle boundaries.

\section{Acknowledgement}

This work was supported in part by the Global COE Program "Materials Integration (International Center of Education and Research), Tohoku University", MEXT, Japan. 


\section{REFERENCES}

1) J. Pan, Q. Chen, N. Li and L. Liu: J. Alloy. Compd. 463 (2008) 246249.

2) J. S. C. Jang, L. J. Chang, T. H. Hung, J. C. Huang and C. T. Liu: Intermetallics 14 (2006) 951-956.

3) Y. L. Gao, J. Shen, J. F. Sun, G. Wang, D. W. Xing, H. Z. Xian and B. D. Zhou: Mater. Lett. 57 (2003) 1894-1898.

4) N. Nishiyama and A. Inoue: Intermetallics 10 (2002) 1141-1147.

5) A. Inoue, B. L. Shen and C. T. Chang: Acta Mater. 52 (2004) 40934099 .

6) A. Inoue, B. L. Shen and C. T. Chang: Intermetallics 14 (2006) 936-
944.

7) R. Watanabe, H. Kimura, H. Kato and A. Inoue: J. Jpn. Soc. Powder Powder Metall. 54 (2007) 761-767.

8) H. Kato, T. Wada, M. Hasegawa, J. Saida, A. Inoue and H. S. Chen: Scr. Mater. 54 (2006) 2023-2027.

9) G. Xie, D. V. Louzguine-Luzgin, H. Kimura and A. Inoue: Appl. Phys. Lett. 90 (2007) 241902.

10) T. S. Kim, J. K. Lee, H. J. Kim and J. C. Bae: Mater. Sci. Eng. A 402 (2005) 228-233.

11) A. Hirata, Y. Hirotsu, K. Amiya, N. Nishiyama and A. Inoue: Intermetallics 16 (2008) 491-497.

12) A. Inoue, B. Shen and A. Takeuchi: Mater. Trans. 47 (2006) 12751285. 